\title{
Association of glycosylated hemoglobin (HbA1c) levels with Iinsulin resistance in obese children.
}

\author{
Zehra Esra Önal, Vildan Atasayan, Tamay Gürbüz, Evrim Hepkaya, Çağatay Nuhoğlu,
}

Haydarpaşa Numune Training and Research Hospital, Department of Pediatrics, Istanbul, Turkey.

\begin{abstract}
Objectives: We investigated the relationship between insulin resistance reflected by homeostasis model assessment (HOMAIR) index and serum HbA1c levels of obese children.

Material and Methods: This study included 70 obese and 60 normal weight healthy children between the ages of 3 and 15 . Anthropometric measures and biochemical tests (fasting glucose, fasting insulin, HbA1c) were performed on all subjects. Plasma glucose levels were measured by the glucose oxidase method. Plasma insulin concentrations were measured by radioimmunoassay (RIA). HOMA-IR index was used to estimate insulin resistance. A cut-off HOMA-IR level of $\geq 2.5$ was accepted. The HbA1c analysis was performed using high-pressure liquid chromatography. The statistical analysis was performed using SPSS 5. Student's unpaired t-test and the Mann-Whitney U test were used to determine statistical significance.

Results: Gender distribution did not reveal significant difference among the obese (F: 48.6\%, M: 51.4\%) and the non-obese (F: 46.7\%, M: 53.3\%) groups. The mean age value was significantly higher in the obese group (10.09 \pm 3.09$)(\mathrm{p}>0.005)$ than the non-obese group $(8.31 \pm 3.14)(\mathrm{p}<0.05)$. The mean value of body mass index (BMI) was $25.55 \pm 4.3$ in the obese group and $16.63 \pm 2.3$ in the non-obese group. The mean HOMA-IR values of obese group $(2.84 \pm 1.77)$ was significantly higher than the non-obese group $(1.50 \pm 0.95)(\mathrm{p}<0.005)$. Insulin resistance was significantly higher in the obese group. Subjects with HOMA-IR $\geq 2.5$ levels in the obese group had significantly higher HbA1c values than those with HOMA-IR $<2.5$ levels.

Conclusions: High HbA1c levels in obese children can be used as a screening tool to detect insulin sensitivity and resistance at an early stage.
\end{abstract}

Key words: children, HbA1c protein, insulin resistance, obesity.

DOI: http://dx.doi.org/10.4314/ahs.v14i3.6

\section{Introduction}

The earlier onset of obesity in children may cause a longer period of insulin resistance, which may explain the concomitant earlier onset of impaired glucose tolerance in children and adolescents. In this study, we determined the validity of $\mathrm{HbA1c}$ screening to identify obese children at high risk for insulin resistance and diabetes at an early stage. We aimed to evaluate the

\section{Corresponding author: \\ Zehra Esra Önal, MD \\ Haydarpasa Numune Training and \\ Research Hospital \\ Department of Pediatrics, Tibbiye \\ Caddesi, No: 40 \\ Selimiye, Uskudar 34668 \\ Istanbul, Turkey. \\ Phone: +90 2164144502 \\ Fax: +90 2163360665 \\ E-mail: zehraesraonal@gmail.com}

association of HOMA-IR and HbA1c levels in obese and non-obese children to assess insulin resistance, a condition that can cause diabetes mellitus and metabolic syndrome in adults.

\section{Material and Methods}

The subjects included 70 obese and 60 non-obese children (130 children in total) between 3 and 15 years old, who were taken to our clinic by the symptomatology of upper respiratory tract infection, between January and December of 2012. Known genetic causes of obesity and chronic illnesses were excluded after having underwent consultations by pediatric endocrinology department. Obesity was defined as a body mass index (BMI) $\geq 95$ th percentile for age and sex, according to the growth references for Turkish children.

Parents were required to sign a written consent to allow their children to participate in this study. All children reported by parents to be suffering from metabolic, endocrine, liver, or kidney disorders were excluded. The study was approved by Local Ethic Committee. 
Height was measured to the last millimeter using a portable stadiometer, and weight was recorded to the nearest $0.11 \mathrm{~kg}$ using a standardized electronic digital scale. Using the measurements, BMI (weight in kilograms divided by the square of the height in meters: $\left.\mathrm{kg} / \mathrm{m}^{2}\right)$ was then computed.

Fasting (12 h) venous blood samples were collected by venipuncture into vacutainer tubes. Once centrifuged, the fractions were separated and frozen at $-70{ }^{\circ} \mathrm{C}$. Plasma glucose levels were measured by the glucose oxidase method with an RA-1000 auto analyzer. Serum insulin concentrations were measured by radioimmunoassay (RIA) using a commercial kit. Insulin resistance was estimated by using the homeostasis model assessment for insulin resistance (HOMA: fasting insulin $[\mathrm{mU} / \mathrm{ml}] \times$ fasting glucose $[\mathrm{mmol} / \mathrm{l}] /$ 22.5). The HbA1c analysis was performed using highpressure liquid chromatography on a Bio-Rad Variant II instrument. All serum samples were stored at $-80{ }^{\circ} \mathrm{C}$ until analysis.

The statistical analysis was performed using SPSS $/ 5$. Student's unpaired t-test (parametric measures) and the Mann-Whitney U test (nonparametric measures) were used to determine statistical significance. The correlation analysis was performed by using Pearson's correlation coefficients. To compare means across different glycemic categories, one-way ANOVA analysis was utilized. $\mathrm{P}$ values $<0.05$ were considered to be statistically significant.

\section{Results}

There was no significant difference between the gender ratios in the obese (Female: 48.6\% / Male: 51.4\%) and in the non-obese (F: 46.7\% / M: 53.3\%) groups. The mean age in the obese group (10.09 \pm 3.09 years) was significantly higher than that in the non-obese group (8.31 \pm 3.14$)(\mathrm{P}<0.05)$. The mean age distribution of the children in the obese and non-obese groups was significantly different. The mean value of the BMI was $25.55 \pm 4.30$ in the obese group and $16.63 \pm 2.3$ in the non-obese group.

The mean value of the HOMA-IR level in the obese group $(2.84 \pm 1.77)$ was significantly higher than that in the non-obese group $(1.5 \pm 0.95)(\mathrm{P}<0.05)$. The percentage of patients with HOMA-IR $\geq 2.5$ was $44.3 \%$ in obese group and $16.7 \%$ in the non obese group (table 1).

Table 1: HOMA-IR values for the obese and non-obese groups

\begin{tabular}{|c|c|c|c|c|c|c|}
\hline & & \multicolumn{2}{|c|}{ Obese Group } & \multicolumn{2}{|c|}{ Non-obese Group } & \multirow[b]{2}{*}{$\mathrm{p}$} \\
\hline & & me & $n-\%$ & $\mathrm{me}$ & $n-\%$ & \\
\hline \multicolumn{2}{|l|}{ HOMA-IR } & \multicolumn{2}{|c|}{$2.84 \pm 1.77$} & \multicolumn{2}{|c|}{$1.50 \pm 0.95$} & $0.000^{* *}$ \\
\hline \multirow[b]{2}{*}{ HOMA-IR } & $<2.5$ & 39 & $55.7 \%$ & 50 & $83.3 \%$ & \multirow[b]{2}{*}{$0.000^{*}$} \\
\hline & $\geq 2.5$ & 31 & $44.3 \%$ & 10 & $16.7 \%$ & \\
\hline
\end{tabular}

SD: Standard deviation; * Chi-Square Test; **Mann-Whitney U Test,

Insulin resistance was significantly higher in the obese group $(\mathrm{P}<0.05)$. There was no statistically significant difference between the HbA1c values of the obese (5.46 \pm 0.5$)$ and non obese groups (5.36 \pm 0.52$)$. Serum fasting glucose levels were not significantly different in the obese $(87.99 \pm 7.17)$ and non-obese groups (86.15 \pm 9.22$)$. The serum fasting insulin level of the obese group $(12.97 \pm 7.91)$ was significantly higher than that of the non-obese group $(6.8 \pm 3.93)(\mathrm{p}<0.05)$ (table 2). 
Table 2: Glucose, Insulin, and HbA1c levels of the obese and non-obese groups

\begin{tabular}{llll}
\hline & \multicolumn{2}{l}{ Obese Group } & \multicolumn{2}{l}{ Non-obese Group } & \multirow{2}{*}{$\mathrm{p}$} \\
\cline { 2 - 3 } & mean $\pm \mathrm{SD} / \mathrm{n}-\%$ & mean $\pm \mathrm{SD} / \mathrm{n}-\%$ & \\
\hline HbA1c $(\%)$ & $5.46 \pm 0.50$ & $5.38 \pm 0.52$ & $0.451^{*}$ \\
Glucose $(\mathrm{mg} / \mathrm{dl})$ & $87.99 \pm 7.17$ & $86.15 \pm 9.22$ & $0.205^{*}$ \\
Insulin $(\mathrm{mIU} / \mathrm{ml})$ & $12.97 \pm 7.91$ & $6.80 \pm 3.93$ & $0.000^{*}$ \\
\hline
\end{tabular}

SD: Standard deviation; *Student t Test

There was a positive correlation between BMI and HOMA-IR, HbA1c, and insulin levels. There was no significant correlation between BMI and glucose levels.

In the obese group, there was no significant gender difference between the children who had HOMA-IR $<2.5$ and HOMA-IR $\geq 2.5$. In the obese group, the mean age distribution was significantly higher for children with HOMA-IR $\geq 2.5$ than for those with HOMA-IR $<2.5(\mathrm{P}<0.05)$.

In the obese group, the HbA1c, insulin, and glucose levels were significantly higher for children with HOMA-IR $\geq 2.5(5.46 \pm 0.27)$ than for those with HOMA-IR $<2.5(\mathrm{P}<0.05)$ (table 3).

Table 3: Evaluation of BMI, HbA1c, and glucose and insulin levels among the obese group with HOMA-IR $\geq 2.5$ and HOMA-IR $<2.5$

\begin{tabular}{llll}
\hline \multirow{2}{*}{ Obese Group } & HOMA-IR $<2.5$ & HOMA-IR $\geq 2.5$ & \\
\cline { 2 - 3 } & mean \pm SD & mean \pm SD & \\
\hline HbA1c $(\%)$ & $5.43 \pm 0.18$ & $5.46 \pm 0.27$ & $0.000^{*}$ \\
Glucose $(\mathrm{mg} / \mathrm{dl})$ & $86.13 \pm 7.77$ & $90.32 \pm 5.64$ & $0.016^{*}$ \\
Insulin $(\mathrm{mIU} / \mathrm{ml})$ & $7.77 \pm 2.90$ & $19.52 \pm 7.34$ & $0.000^{*}$ \\
BMI $\left(\mathrm{kg} / \mathrm{m}^{2}\right)$ & $23.45 \pm 3.23$ & $28.17 \pm 4.07$ & $0.476^{*}$
\end{tabular}

SD: standard deviation; *Mann-Whitney U Test

In the non-obese group, the glucose and insulin levels were significantly higher for children with
HOMA-IR $\geq 2.5$ than for those with HOMA-IR $<2.5$ (table 4). 
Table 4: Evaluation of BMI, HbA1c, and glucose and insulin levels among the non-obese group with HOMA-IR $\geq 2.5$ and HOMA-IR $<2.5$

\begin{tabular}{llll}
\hline \multirow{2}{*}{ Non-obese Group } & HOMA-IR $<2.5$ & HOMA-IR $\geq 2.5$ & \\
\cline { 2 - 3 } & mean $\pm \mathrm{SD}$ & mean $\pm \mathrm{SD}$ & $\mathrm{p}$ \\
\hline HbA1c $(\%)$ & $5.38 \pm 0.20$ & $5.30 \pm 0.36$ & $0.606^{*}$ \\
Glucose $(\mathrm{mg} / \mathrm{dl})$ & $84.84 \pm 9.16$ & $92.70 \pm 6.60$ & $0.013^{*}$ \\
Insulin $(\mathrm{mIU} / \mathrm{ml})$ & $5.42 \pm 2.49$ & $13.70 \pm 1.95$ & $0.000^{*}$ \\
BMI $\left(\mathrm{kg} / \mathrm{m}^{2}\right)$ & $16.52 \pm 2.15$ & $17.18 \pm 3.01$ & $0.695^{*}$
\end{tabular}

SD: standard deviation; *Mann-Whitney U Test

But there was no positive correlation between HOMA-IR and HbA1c levels in the non-obese group.

The HOMA-IR measurements were significantly higher in children with HbA1c levels $\geq 5.5(2.71 \pm 1.63)$ than in those with HbA1c levels $<5.5(2.08 \pm 1.56)$ $(\mathrm{p}<0.05)$ (table 5).

Table 5: Evaluation of HOMA-IR levels between the groups with HbA1c $\leq 5.5$ and $\mathrm{HbA} 1 \mathrm{c}>5.5$

\begin{tabular}{llll}
\hline & HbA1c $\leq 5.5$ & HbA1c $>5.5$ & \\
mean \pm SD & mean \pm SD & p \\
\hline HOMA-IR & $2.08 \pm 1.56$ & $2.71 \pm 1.63$ & $0.035^{*}$
\end{tabular}

SD: standard deviation, * Mann-Whitney U Test

\section{Discussion}

The prevalence of obesity in children and adolescents has reached epidemic proportions in both developed and developing countries $\square$ (1). Insulin resistance in adults has been recognized for decades as a cardinal feature in the development of type 2 diabetes and has been associated with obesity, metabolic syndrome, hypertension, and heart disease $(2,3)$. Obesity Research Clinical Practice also clear that insulin resistance is significantly related to obesity and cardio metabolic risk in children $(4,5)$.

Because an earlier onset of obesity in children is responsible for lengthening the insulin-resistant status of obese children, recognition of impaired glucose tolerance in children is important. Previous studies have reported that 13-14\% of children between 6 and 10 years old and adolescents between 12 and 19 years old were overweight. The epidemic percentage of obese young people reached $15.5 \%$ during the years 1999 $2001(6)$. Subjects who had a BMI of $28 \mathrm{~kg} / \mathrm{m}^{2}$ showed a marked increase in the frequency of cardiovascular risk factors such as high blood pressure, dyslipidemia, and insulin resistance (7). It has been reported that childhood obesity can lead to complications involving insulin resistance in adulthood, so it is important to prevent obesity in the young at-risk population (8).

HOMA, the most widely used surrogate measure in children, is highly correlated with fasting insulin ( $\mathrm{r}$ $\geq 0.95$ ) in children (9). Fasting glucose is maintained within a narrow range among obese children and those with abnormal glucose tolerance $(10,11)$.

In a study at the University Aichi in Japan, the fasting insulin levels of 10-13 year old girls were significantly higher $(8.83 \pm 3.74 \mathrm{mIU} / \mathrm{ml})$ than those of the boys $(7.09 \pm 3.27 \mathrm{mIU} / \mathrm{ml})(\mathrm{p}<0.01)$. The HOMAIR levels of Japanese girls $(2.01 \pm 0.89)$ 10-13 years old were significantly higher than those of the boys $(1.67 \pm 0.81)(p<0.05)$. German girls $5-17$ years old had a higher HOMA-IR mean value of 3.13 while boys from a similar cohort had a HOMA-IR level of 2.64). The mean HOMA-IR level of 7-10 year old Brazilian girls was $3.8 \pm 2.3$, which was higher than that of the boys in the study $(2.6 \pm 1.3)(11,12)$.

In their study, Hoffman et al. reported that girls 
were less sensitive to insulin, so they compensated by increasing insulin secretions. This decreased sensitivity is why girls carry a higher insulin resistance risk than boys (13).

In a study in Japan, although HbA1c levels and fasting glucose levels did not show a correlation with the BMI values, fasting insulin and HOMA-IR levels were significantly higher in overweight children. The mean value of the HOMA-IR levels was 2.51 1 1.01 (showing insulin resistance) in the overweight group, and the percentage of subjects with HOMA-IR levels $\geq 2.5$ was $46.8 \%$. However, in the other groups, which included normal weight children, the percentage was $13-20 \%$. Japanese overweight children showed a tendency toward high insulin resistance (14).

In our study, the mean value of the HOMA-IR levels was higherin the obese group than in the non-obese group. The percentage of subjects with HOMA-IR $\geq 2.5$ was $44.3 \%$ in the obese group, while the percentage was only $16.7 \%$ in the non-obese group. Insulin resistance and fasting insulin levels were significantly higher in the obese group compared with the non-obese group. Our findings are similar to previous studies in the literature.

Valerio et al. from Italy reported that among 3-16 year old children, age or pubertal stage and BMI were associated with insulin resistance (HOMA-IR). The positive correlation with age, with pubertal stage in particular, is well documented because puberty is as sociated with a transient reduction of 25-30\% in insulin sensitivity that mainly occurs in mid-puberty. Valerio's study demonstrated that the mean age of patients with HOMA-IR levels $\geq 2.5$ was higher in the obese group than the mean age of patients with HOMA-IR $\leq 2.5$ in the non-obese group. In the obese group, $80 \%$ of the children were in the pubertal age range of $8-12$ years old, and in the non-obese group, $56 \%$ of the children were in the 8-12 year old pubertal stage (15). These findings are similar to those from our study, which show that HOMA-IR levels correlate with age, particularly the pubertal stage. In our study, the percentage of 8-12 years old children was $14.3 \%$ in the obese group and $51.6 \%$ in the non-obese group. The HOMA-IR levels of the obese group were significantly higher than those of the non-obese group. Most of the children in the obese group who had a high HOMA-IR level were in the pubertal stage. During puberty, there is a $25-30 \%$ decline in insulin sensitivity, which is recovered when pubertal development is complete (16).

Although HbA1c is used to diagnose type II diabetes, it is unknown whether elevated HbA1c levels within a non-diabetic range can reliably reflect insulin resistance or impairment of insulin secretion in individuals not being treated for diabetes. A study performed in Toranoman Hospital in Japan, published in Diabetic Medicine in 2012, demonstrated that elevated $\mathrm{HbA} 1 \mathrm{c}$ levels within a non-diabetic range were associated with impaired insulin secretion, even without escalating insulin resistance. Elevated $\mathrm{HbA} 1 \mathrm{c}$ levels $\geq 5.9 \%$ were associated with substantial reductions in insulin secretion and insulin sensitivity and in $\beta$-cell dysfunction in Japanese individuals. Moreover, impaired early phase insulin secretion and relative $\beta$-cell dysfunction were suggested to already exist among individuals with high normal HbA1c (5.4-5.8\%) levels even when insulin resistance was not increased (17).

In the obese group in our study, children with HOMA-IR levels $\geq 2.5$ showed significantly higher HbA1c values than those with HOMA-IR levels $<2$. However, in the non obese group, there was no significant difference in the HbA1c levels of those with HOMA-IR $\geq 2.5$ and those with HOMA-IR $<2.5$. These results demonstrate that insulin resistance related to obesity plays a major role in the development of diabetes mellitus.

HbA1c has the potential to reflect the history of mean insulin sensitivity over the preceding weeks or months, and it serves as a marker of insulin sensitivity in children who have a normal glucose tolerance (18). $\mathrm{HbA1c}$ levels can be used as a diagnostic tool for the early detection of insulin resistance.

Since we found a positive correlation between $\mathrm{HbA1c}$ and HOMA-IR levels only in the obese group, we suggest using $\mathrm{HbA} 1 \mathrm{c}$ levels as a simple and cheaper marker only in obese children with relatively high insulin sensitivity. Insulin resistance can be evaluated by screening HOMA-IR levels in obese children. But in higher HbA1c levels within the ranges of 5.5-6\%, we determined a correlation between HOMA-IR and HbA1c levels. We suggest firstly screening HbA1c levels, a cheaper and easy method ( no need to fasting) in order to evaluate insulin sensitivity in obese children. Comprehensive studies that include more subjects can be used to confirm this assertion in the future.

Insulin resistance, which is the component of metabolic syndrome, is more commonly seen in obese children, compared to non-obese ones. It is important to identify insulin resistance at an early stage. HbA1c screening can be used as a cheaper and simple ( no need to fasting ) method in order to evaluate insulin resistance in obese children. 


\section{References}

1. Shalitin S, Abrahami M, Lilos P, Phillip M (2005). Insulin resistance and impaired glucose tolerance in obese children and adolescents referred to a tertiarycare center in Israel. Int J Obes (Lond), 29(6):571-8.

2. Reaven GM:Banting lecture (1988). Role of insulin resistance in human disease. Diabetes, 37(12):1595607.

3. Cameron AS, Shaw JE, Zimmet PZ (2004). The metabolic syndrome: prevalence in worldwide populations. Endocrinol Metab Clin North Am, 33(2):351-75.

4. Invitti C, Gilardini L, Viberti G (2002). Impaired glucose tolerance in obese children and adolescents. $\mathrm{N}$ Engl J Med, 347(4):290-2.

5. Ten S, Maclaren N (2004). Insulin resistance syndrome in children. The Journal of Clin Endocrinol Metab, 89(6):2526-39.

6. Willett WC, Stampfer M, Manson J, VanItallie T (1991). New weight guidelines for Americans: justified or injudicious? Am J Clin Nutr, 53(5):1102-3.

7. Long SD, O’Brien K, MacDonald KG Jr, LeggettFrazier N, Swanson MS, Pories WJ, et al. (1994). Weight loss in severely obese subjects prevents the progression of impaired glucose tolerance to type II diabetes. A longitudinal interventional study. Diabetes Care, 17(5):372-5.

8. Herder C, Schneitler S, Rathmann W, Haastert B, Schneitler H, Winkler H, et al. (2007). Low- grade inflammation, obesity, and insulin resistance in adolescents. J Clin Endocrinol Metab, 92(12):4569-74.

9. Schwartz B, Jacobs JR DR, Moran A, Steinberger J, Hong CP, Sinaiko AR (2008). Measurement of insulin sensitivity in children: comparison between the euglycemic-hyperinsulinemic clamp and surrogate measures. Diabetes Care, 31(4):783-8.

10. Weiss R, Dziura J, Burgert TS, Tomborlane WV, Taksali SE, Yeckel CW,et al. (2004). Obesity and the metabolic syndrome in children and adolescents. N Engl J Med, 350(23):2362-74.

11. Sinha R, Fisch G, Teague B, Tamborlane WV, Banyas B, Allen K, et al. (2002): Prevelance of impaired glucose tolerance among children and adolescents with marked obesity. N Engl J Med., 14;346(11):802-10.

12. Ferreira AP, Oliveira CE, Franca NM (2007). Metabolic syndrome and risk factors for cardiovascular disease in obese children: the relationship with insulin resistance (HOMA-IR). J Pediatr (RioJ), 83(1):21-6.

13. Hoffman RP, Vicini P, Sivitz WI, Cobelli C (2000): Pubertal adolescent male-female differences in insulin sensitivity and glucose effectiveness determined by the one compartment minimal model. Pediatr Res.48(3): 384-8.

14. Fujii C, Sakakibara H (2012). Association between insulin resistance, cardiovascular risk factors and overweight in Japanese school children.Obesity Research \&Clinical Practice, 6:e1-e90.

15. Valerio G, Licenziati MR, Iannuzzi A, Franzese A, Siani P, Riccardi G, et al. (2006).Insulin Resistance and impaired glucose resistance in obese children and adolescent from southern Italy. Nutr Metab Cardiovasc Dis, 16(4):279-84.

16. Goran MI, Gower BA (2001). Longitudinal study on pubertal insulin resistance. Diabetes, 50(11):244450 .

17. Heianza Y, Arase Y, Fujihara K, Tsuji H, Saito K, Hsieh SD, et al. (2012). High normal HbA1c levels were associated with impaired insulin secretion without escalating insulin resistance in Japanese individuals: the Toranomon Hospital Health Management Center Study 8. Diabet Med, 29(10):1285-90.

18. Borai A, Livingstone C, Abdelaal F, Bawazeer A, Keti V, Ferns G (2011): The relationship between glycosylated heamoglobin (HbA1c) and measures of insulin resistance across a range of glucose tolerance. Scand J Clin Lab Invest, 71(2):168-72. 